\title{
Integral spinor norms in dyadic local fields II
}

\author{
by
}

FEI Xu (Hefei)

In the previous paper $[\mathrm{X}]$ we have generalized the results of $[\mathrm{BD}]$ to a dyadic local field with $e=\operatorname{ord} 2=2$. In the present paper we generalize these results to an arbitrary dyadic local field, and we also point out that the bound for ord $(d L)$ is the best possible. The results obtained are applied to improve the sufficient condition for the class number of an indefinite quadratic form over the ring of integers of a number field to be a divisor of the class number of the field, which is analogous to Satz 5 of $[\mathrm{K}]$.

Here we adopt the notations from $[\mathrm{O}]$ and $[\mathrm{X}]$. In particular, $F$ denotes a dyadic local field, $\vartheta$ the ring of integers in $F, p$ the maximal ideal of $\vartheta$, $U$ the group of units in $\vartheta, e=$ ord 2 the ramification index of 2 in $F, \pi$ a fixed prime element in $F, D($,$) the quadratic defect function, \Delta$ a fixed unit of quadratic defect $4 \vartheta, V$ a regular quadratic space over $F$ with associated symmetric bilinear form $B(x, y), L$ a lattice on $V, d L$ the determinant of $L$, $O^{+}(V)$ the group of rotations on $V, O^{+}(L)$ the corresponding subgroup of units of $L$, and $\theta($, ) the spinor norm function. We use the symbol $\langle a, b, c, \ldots\rangle$ for lattices, and $[a, b, c, \ldots]$ for spaces.

Lemma 1. For any $i \geq 1,1+p^{i}$ is generated by $1+\lambda \pi^{i}$ with $\lambda \in U$.

Proof. This follows from the identity

$$
\left(1+\sigma \pi^{k+1}\right)=\left(1+\pi^{k}\right)\left(1+\left(1+\pi^{k}\right)^{-1}(\sigma \pi-1) \pi^{k}\right) .
$$

Lemma 2. Suppose $s L \subseteq \vartheta$ and $\operatorname{rank} L \geq 3$ and $e \geq 3$. If $\operatorname{ord}(d L) \leq 3$ then $\theta\left(O^{+}(L)\right) \supseteq U \dot{F}^{2}$.

Proof. Let $L=L_{1} \perp \ldots \perp L_{t}$ be a Jordan splitting of $L$. We assume $t \geq 2$ and $\operatorname{rank} L_{i} \leq 2, i=1, \ldots, t$. Since $\operatorname{ord}(d L) \leq 3, t \leq 3$. We consider several cases.

(1) $L=L_{1} \perp L_{2}$ where $L_{1}$ is unimodular with $\operatorname{rank} L_{1}=2$ and $L_{2}=\vartheta x_{2}$ with $Q\left(x_{2}\right)=\varepsilon_{2} \pi$ and $\varepsilon_{2} \in U$. Put $L_{1} \cong A\left(a_{1},-a_{1}^{-1} \delta_{1}\right)$ with the base $\left\{x_{1}, y_{1}\right\}$ and $0 \leq$ ord $a_{1} \leq e$ and $D\left(1+\delta_{1}\right)=\delta_{1} \vartheta$.

If $\operatorname{ord}\left(-a_{1}^{-1} \delta_{1}\right)<e$, then ord $a_{1} \equiv \operatorname{ord}\left(-a_{1}^{-1} \delta_{1}\right)+1 \bmod 2$. 
When ord $a_{1}$ is even, take $K=\vartheta x_{1} \perp \vartheta x_{2}$. Note that any maximal vector of $K$ gives rise to a symmetry of $L$. So $\theta\left(O^{+}(L)\right) \supseteq Q\left(\left[1, \dot{a}_{1} \varepsilon_{2} \pi\right]\right)$ which does not contain $\Delta$, but $\Delta$ is in $\theta\left(O^{+}\left(L_{1}\right)\right)$ by $[\mathrm{H}]$. Therefore $\theta\left(O^{+}(L)\right)=\dot{F}$.

When ord $a_{1}$ is odd, then ord $\left(-a_{1}^{-1} \delta_{1}\right)$ is even. Take $K=\vartheta y_{1} \perp \vartheta x_{2}$, and $\theta\left(O^{+}(L)\right)=\dot{F}$ by the same arguments as above.

If ord $\left(-a_{1}^{-1} \delta_{1}\right) \geq e$, write $a_{1}=\varepsilon_{1} \pi^{r_{1}}$ and $-\varepsilon_{1} \varepsilon_{2}^{-1}=\eta^{2}+\sigma \pi^{d}$ where $d$ is an odd integer or $d \geq 2 e$.

When $r_{1}$ is odd, consider a unimodular lattice $\bar{L}_{1}=\vartheta\left(x_{1}+\eta \pi^{\left(r_{1}-1\right) / 2} x_{2}\right)$ $+\vartheta y_{1}$ which splits $L$. Write $L=\bar{L}_{1} \perp \vartheta \bar{x}_{2}$ with $Q\left(\bar{x}_{2}\right)=\bar{\varepsilon}_{2} \pi$. Note

$$
\operatorname{ord}\left(Q\left(x_{1}+\eta \pi^{\left(r_{1}-1\right) / 2} x_{2}\right)\right)=r_{1}+d .
$$

If $r_{1}+d \geq e$, then $\bar{L}_{1} \cong A(0,0)$ or $A(2,2 \varrho)$ by [O, 93:11]. Therefore $\theta\left(O^{+}(L)\right) \supseteq \theta\left(O^{+}\left(\bar{L}_{1}\right)\right)=U \dot{F}^{2}$ by [H, Lemma 1]. Otherwise, $r_{1}+d<e$ and $r_{1}+d$ is even. Take $K=\vartheta\left(x_{1}+\eta \pi^{\left(r_{1}-1\right) / 2} x_{2}\right) \perp \vartheta \bar{x}_{2}$. Therefore $\theta\left(O^{+}(L)\right)$ $=\dot{F}$.

When $r_{1}$ is even, take $K=\vartheta x_{1} \perp \vartheta x_{2}$. So $\theta\left(O^{+}(L)\right)=\dot{F}$.

(2) $L=L_{1} \perp L_{2}$ where $L_{1}$ is unimodular with $\operatorname{rank} L_{1}=2$ and $L_{2}=\vartheta x_{2}$ with $Q\left(x_{2}\right)=\varepsilon_{2} \pi^{2}$ and $\varepsilon_{2} \in U$. By the arguments similar to Case (1), we only need to consider $L_{1} \cong A\left(\varepsilon_{1},-\varepsilon_{1}^{-1} \delta_{1}\right)$ with the base $\left\{x_{1}, y_{1}\right\}$ where $\varepsilon_{1}$ is in $U, D\left(1+\delta_{1}\right)=\delta_{1} \vartheta$ and $\operatorname{ord}\left(-\varepsilon_{1}^{-1} \delta_{1}\right)>e$. Put $D\left(\varepsilon_{1} \varepsilon_{2}\right)=p^{t}$ with $1 \leq t \leq 2 e$ or $t=\infty$.

If $t \leq e-1$, then $D\left(-\varepsilon_{1} \varepsilon_{2}\right)=D\left(\varepsilon_{1} \varepsilon_{2}\right)=p^{t}$. Take $K=\vartheta x_{1} \perp \vartheta x_{2}$. Note that any maximal vector of $K$ gives rise to a symmetry of $L$, so $\theta\left(O^{+}(L)\right) \supseteq$ $Q\left(\left[1, \dot{\varepsilon}_{1} \varepsilon_{2}\right]\right)$. By [H, Lemma 3], there exists $\eta$ in $U$ such that $\left(\eta,-\varepsilon_{1} \varepsilon_{2}\right)=-1$ with $D(\eta)=p^{2 e-t}$. Since $2 e-t \geq e+1, \eta$ is in $\theta\left(O^{+}\left(L_{1}\right)\right)$ by [H, Lemma 2]. Therefore $\theta\left(O^{+}(L)\right)=\dot{F}$.

If $t>e-1$, write $\varepsilon_{2}^{-1} \varepsilon_{1}=\xi^{2}+\sigma \pi^{t}$ where $\xi$ and $\sigma$ are in $U$.

When $e$ is odd, there exists $u$ in $\vartheta$ such that

$$
\lambda+2 \pi^{-e} \xi\left(\pi^{(e-1) / 2}-\xi\right)-\sigma \pi^{t-e}-2 \pi^{-e} \varepsilon_{2}^{-1} u+\varepsilon_{1}^{-1} \varepsilon_{2}^{-1} \delta_{1} \pi^{-e} u^{2}=0
$$

for any $\lambda \in \vartheta$ by Hensel's Lemma. Put $z=\pi x_{1}+\pi u y_{1}+\left(\pi^{(e-1) / 2}-\xi\right) x_{2} \in L$, and

$$
\begin{aligned}
Q(z)= & \pi^{2} \varepsilon_{1}+\pi^{2} u^{2}\left(-\varepsilon_{1}^{-1} \delta_{1}\right)+2 \pi^{2} u+\left(\pi^{(e-1) / 2}-\xi\right)^{2} \varepsilon_{2} \pi^{2} \\
= & \varepsilon_{2} \pi^{2}\left(\xi^{2}+\sigma \pi^{t}+2 \varepsilon_{2}^{-1} u+\left(\pi^{(e-1) / 2}-\xi\right)^{2}-\left(\varepsilon_{1} \varepsilon_{2}\right)^{-1} \delta_{1} u^{2}\right) \\
= & \varepsilon_{2} \pi^{2}\left(\pi^{e-1}-2 \xi\left(\pi^{(e-1) / 2}-\xi\right)+\sigma \pi^{t}+2 \varepsilon_{2}^{-1} u-\left(\varepsilon_{1} \varepsilon_{2}\right)^{-1} \delta_{1} u^{2}\right) \\
= & \varepsilon_{2} \pi^{e+1}\left(1+\pi\left(-2 \pi^{-e} \xi\left(\pi^{(e-1) / 2}-\xi\right)\right.\right. \\
& \left.\left.+\sigma \pi^{t-e}+2 \pi^{-e} \varepsilon_{2}^{-1} u-\left(\varepsilon_{1} \varepsilon_{2}\right)^{-1} \delta_{1} \pi^{-e} u^{2}\right)\right) \\
= & \varepsilon_{2} \pi^{e+1}(1+\lambda \pi) .
\end{aligned}
$$

So $\tau_{z}$ is in $O(L)$ and $\theta\left(O^{+}(L)\right) \supseteq U \dot{F}^{2}$. 
When $e$ is even, there exists $u$ in $\vartheta$ such that

$\lambda+2 \pi^{1-e} \xi\left(\pi^{(e-2) / 2}-\xi\right)-\sigma \pi^{t-e+1}-2 \pi^{-e} \varepsilon_{2}^{-1} u+\left(\varepsilon_{1} \varepsilon_{2}\right)^{-1} \delta_{1} \pi^{-e-1} u^{2}=0$

for any $\lambda \in \vartheta$ by Hensel's Lemma if $\operatorname{ord}\left(\delta_{1}\right) \geq e+2$. Put

$$
z=\pi x_{1}+u y_{1}+\left(\pi^{(e-2) / 2}-\xi\right) x_{2} \in L .
$$

Since $Q(z)=\varepsilon_{2} \pi^{e}(1+\lambda \pi)$ by a direct computation, $\tau_{z}$ is in $O(L)$ and $\theta\left(O^{+}(L)\right) \supseteq U \dot{F}^{2}$.

Now we treat the case of $\operatorname{ord}\left(-\varepsilon_{1}^{-1} \delta_{1}\right)=e+1$. For any $\lambda \in U$, write

$$
\left(-\left(\varepsilon_{1} \varepsilon_{2}\right)^{-1} \delta_{1} \pi^{-e-1}\right)^{-1}=\alpha^{2}+\beta \pi^{d}
$$

where $\alpha$ and $\beta$ are in $U$, and $d \geq 1$. By Hensel's Lemma, there exists $u$ in $\vartheta$ such that

$$
\begin{aligned}
\left(\varepsilon_{1} \varepsilon_{2}\right)^{-1}\left(\delta_{1} \pi^{-e-1}\right) \beta \pi^{d-1} & \\
& +\left(2 \pi^{-e} \alpha \varepsilon_{2}^{-1}-2 \xi \pi^{-e / 2}\right) u+\left(\sigma \pi^{t+2-e}+2 \xi^{2} \pi^{2-e}\right) u^{2}=0 .
\end{aligned}
$$

Put $z=\pi^{2} u x_{1}+\alpha y_{1}+\left(\pi^{(e-2) / 2}-\xi \pi u\right) x_{2} \in L$. So $Q(z)=\varepsilon_{2} \pi^{e}(1+\lambda \pi)$ and $\tau_{z}$ is in $O(L)$. Therefore we obtain $\theta\left(O^{+}(L)\right) \supseteq U \dot{F}^{2}$ by Lemma 1 .

(3) $L=L_{1} \perp L_{2}$ where $L_{1}$ is unimodular with $\operatorname{rank} L_{1}=2$ and $L_{2}=\vartheta x_{2}$ with $Q\left(x_{2}\right)=\varepsilon_{2} \pi^{3}$ and $\varepsilon_{2} \in U$. By the arguments similar to Case (1), we only need to consider $L_{1} \cong A\left(\varepsilon_{1} \pi_{1},-\varepsilon_{1}^{-1} \pi^{-1} \delta_{1}\right)$ with the base $\left\{x_{1}, y_{1}\right\}$ where $\varepsilon_{1}$ is in $U, D\left(1+\delta_{1}\right)=\delta_{1} \vartheta$ and $\operatorname{ord}\left(-\varepsilon_{1}^{-1} \pi^{-1} \delta_{1}\right)>e$. Put $D\left(\varepsilon_{1} \varepsilon_{2}\right)=p^{t}$ with $1 \leq t \leq 2 e$ or $t=\infty$.

If $t \leq e-2$, take $K=\vartheta x_{1} \perp \vartheta x_{2}$. By the same arguments as in Case (2), we have $\theta\left(O^{+}(L)\right)=\dot{F}$.

If $t>e-2$, write $\varepsilon_{2}^{-1} \varepsilon_{1}=\xi^{2}+\sigma \pi^{t}$ where $\xi$ and $\sigma$ are in $U$.

When $e$ is even, there exists $u$ in $\vartheta$ such that

$$
\lambda+2 \pi^{1-e} \xi\left(\pi^{(e-2) / 2}-\xi\right)-\sigma \pi^{t-e+1}-2 \pi^{-e} \varepsilon_{2}^{-1} u+\left(\varepsilon_{1} \varepsilon_{2}\right)^{-1} \delta_{1} \pi^{-e-1} u^{2}=0
$$

for any $\lambda \in \vartheta$ by Hensel's Lemma. Put $z=\pi x_{1}+\pi u y_{1}+\left(\pi^{(e-2) / 2}-\xi\right) x_{2} \in L$ and $Q(z)=\varepsilon_{2} \pi^{e+1}(1+\lambda \pi)$; so $\tau_{z}$ is in $O(L)$ and $\theta\left(O^{+}(L)\right) \supseteq U \dot{F}^{2}$.

When $e$ is odd, there exists $u$ in $\vartheta$ such that

$$
\lambda+2 \pi^{2-e} \xi\left(\pi^{(e-3) / 2}-\xi\right)-\sigma \pi^{t-e+2}-2 \pi^{-e} \varepsilon_{2}^{-1} u+\left(\varepsilon_{1} \varepsilon_{2}\right)^{-1} \pi^{-e-2} \delta_{1} u^{2}=0
$$

for any $\lambda \in \vartheta$ by Hensel's Lemma if ord $\left(-\varepsilon_{1}^{-1} \pi^{-1} \delta_{1}\right)>e+1$. Put

$$
z=\pi x_{1}+u y_{1}+\left(\pi^{(e-3) / 2}-\xi\right) x_{2} \in L .
$$

Since $Q(z)=\varepsilon_{2} \pi^{e}(1+\pi \lambda), \tau_{z}$ is in $O(L)$ and $\theta\left(O^{+}(L)\right) \supseteq U \dot{F}^{2}$.

Now we treat the case of $\operatorname{ord}\left(-\varepsilon_{1}^{-1} \pi^{-1} \delta_{1}\right)=e+1$. For any $\lambda \in U$, write

$$
\lambda\left(-\left(\varepsilon_{1} \varepsilon_{2}\right)^{-1} \pi^{-2-e} \delta_{1}\right)^{-1}=\alpha^{2}+\beta \pi^{d}
$$


where $\alpha$ and $\beta$ are in $U$ and $d \geq 1$. By Hensel's Lemma, there exists $u$ in $\vartheta$ such that

$$
\begin{aligned}
\beta \pi^{d}\left(\varepsilon_{1} \varepsilon_{2}\right)^{-1} \pi^{-2-e} \delta_{1}+\left(2 \varepsilon_{2}^{-1} \alpha \pi^{-e}-2 \xi \pi^{(1-e) / 2}\right) u & \\
+\left(\sigma \pi^{t-e+2}+2 \pi^{2-e} \xi^{2}\right) u^{2} & =0 .
\end{aligned}
$$

Put $z=\pi u x_{1}+\alpha y_{1}+\left(\pi^{(e-3) / 2}-\xi u\right) x_{2} \in L$ and $Q(z)=\varepsilon_{2} \pi^{e}(1+\lambda \pi)$. So $\tau_{z}$ is in $O(L)$ and $\theta\left(O^{+}(L)\right) \supseteq U \dot{F}^{2}$ by Lemma 1.

(4) $L=L_{1} \perp L_{2}$ where $L_{1}$ is unimodular with $\operatorname{rank} L_{1}=2$ and $L_{2}$ is $p$-modular with $\operatorname{rank} L_{2}=2$. Write $L_{1} \cong A\left(\varepsilon_{1} \pi^{r_{1}},-\varepsilon_{1}^{-1} \pi^{-r_{1}} \delta_{1}\right)$ with the base $\left\{x_{1}, y_{1}\right\}$ and $0 \leq r_{1} \leq e$ and $D\left(1+\delta_{1}\right)=\delta_{1} \vartheta$. $L_{2} \cong \pi A\left(\varepsilon_{2} \pi^{r_{2}}\right.$, $\left.-\varepsilon_{2}^{-1} \pi^{-r_{2}} \delta_{2}\right)$ with the base $\left\{x_{2}, y_{2}\right\}$ and $0 \leq r_{2} \leq e$ and $D\left(1+\delta_{2}\right)=\delta_{2} \vartheta$.

If $r_{1} \equiv r_{2} \bmod 2$, take $K=\vartheta x_{1} \perp \vartheta x_{2}$. By the same arguments as in Case (1), we obtain $\theta\left(O^{+}(L)\right)=\dot{F}$.

If $r_{1} \equiv r_{2}+1 \bmod 2$, write $-\varepsilon_{1} \varepsilon_{2}^{-1}=\xi^{2}+\sigma \pi^{d}$ with $\xi, \sigma \in U$ and $d \geq 1$.

When ord $\left(-\varepsilon_{1}^{-1} \pi^{-r_{1}} \delta_{1}\right)<e$, take $K=\vartheta y_{1} \perp \vartheta x_{2}$; thus $\theta\left(O^{+}(L)\right)=\dot{F}$.

When ord $\left(-\varepsilon_{2}^{-1} \pi^{-r_{2}} \delta_{2}\right)<e$, take $K=\vartheta x_{1} \perp \vartheta y_{2}$; thus $\theta\left(O^{+}(L)\right)=\dot{F}$.

Otherwise, we take $\bar{L}_{1}=\vartheta\left(x_{1}+\xi \pi^{\left(r_{1}-r_{2}-1\right) / 2} x_{2}\right)+\vartheta y_{1}$ splitting $L$ if $r_{1} \geq r_{2}+1$, or $\bar{L}_{2}=\vartheta\left(x_{2}+\xi^{-1} \pi^{\left(r_{2}-r_{1}-1\right) / 2} x_{1}\right)+\vartheta y_{2}$ splitting $L$ if $r_{1}<r_{2}+1$ by $[\mathrm{O}, 82: 15]$.

When $\operatorname{ord}\left(Q\left(x_{1}+\xi \pi^{\left(r_{1}-r_{2}-1\right) / 2} x_{2}\right)\right)=r_{1}+d \geq e$ or $\operatorname{ord}\left(Q\left(x_{2}+\right.\right.$ $\left.\left.\xi^{-1} \pi^{\left(r_{2}-r_{1}+1\right) / 2} x_{1}\right)\right)=r_{2}+d+1 \geq e$, then $\bar{L}_{1}$ or $\bar{L}_{2} \cong A(0,0)$ or $A(2,2 \varrho)$, and $\theta\left(O^{+}(L)\right) \supseteq \theta\left(O^{+}\left(\bar{L}_{1}\right)\right)=U \dot{F}^{2}$ or $\theta\left(O^{+}\left(\bar{L}_{2}\right)\right)=U \dot{F}^{2}$.

If $\operatorname{ord}\left(Q\left(x_{1}+\xi \pi^{\left(r_{1}-r_{2}-1\right) / 2} x_{2}\right)\right)=r_{1}+d<e$ or $\operatorname{ord}\left(Q\left(x_{2}+\right.\right.$ $\left.\left.\xi^{-1} \pi^{\left(r_{2}-r_{1}+1\right) / 2} x_{1}\right)\right)=r_{2}+d+1<e$, then $L \cong \bar{L}_{1} \perp L_{2}^{\prime}$ or $L_{1}^{\prime} \perp \bar{L}_{2}$ respectively and we repeat the above arguments until we obtain the results as desired.

(5) $L=L_{1} \perp L_{2} \perp L_{3}$ where $L_{1}$ is unimodular with $\operatorname{rank} L_{1}=2$, and $L_{i}=$ $\vartheta x_{i}$ with $Q\left(x_{i}\right) \vartheta=p^{i-1}, i=2,3$. Then $\theta\left(O^{+}(L)\right) \supseteq \theta\left(O^{+}\left(L_{1} \perp L_{2}\right)\right) \supseteq U \dot{F}^{2}$ by Case (1).

(6) $L=L_{1} \perp L_{2}$ with $\operatorname{rank} L_{1}=1$ and $\operatorname{rank} L_{2}=2$. We scale the dual lattice of $L$ by $\pi$ and reduce to Case (1).

(7) $L=L_{1} \perp L_{2} \perp L_{3}$ with $\operatorname{rank} L_{i}=1, i=1,2,3$. So $L_{i}=\vartheta x_{i}$ with $Q\left(x_{i}\right)=p^{i-1}, i=1,2,3$; and $\theta\left(O^{+}(L)\right)=\dot{F}$ by [X, Theorem 3.1].

We point out that the bound ord $(d L) \leq 3$ given in the above lemma cannot be unconditionally improved for any $e \geq 3$ in view of the following example.

Example. Suppose $L \cong A\left(1, \pi^{2 e-1}\right) \perp\left\langle\pi^{4}\right\rangle$ with the base $\{x, y, z\}$ and $e \geq 3$. Then $\theta\left(O^{+}(L)\right) \subseteq\left(1+p^{2}\right) \dot{F}^{2}$.

Proof. First we prove that $O(L)$ is generated by the symmetries of $L$. 
Take $\sigma$ in $O(L)$. Write $\sigma x=a x+b y+c z$. So $1-a^{2}=2 a b+b^{2} \pi^{2 e-1}+c^{2} \pi^{4} \in$ $p^{3}$ and $(1-a) \in p^{2}$. We can assume ord $b \leq 1$, otherwise, instead of $\sigma$ we consider $\tau_{\pi^{[e / 2]} x+y} \sigma$ if necessary and $\tau_{\pi^{[e / 2]} x+y} \in O(L)$. Since $Q(\sigma x-x)=$ $2((1-a)-b), \tau_{\sigma x-x} \in O(L)$. Therefore we assume $\sigma x=x, \sigma y=\alpha x+\beta y+\gamma z$. So

$$
\alpha+\beta=1, \quad \pi^{2 e-1}=\alpha^{2}+2 \alpha \beta+\beta^{2} \pi^{2 e-1}+\gamma^{2} Q(z)
$$

and

$$
Q(\sigma y-y)=2 \alpha\left(-1+\pi^{2 e-1}\right) .
$$

When ord $\alpha \leq 4$, then $\tau_{\sigma y-y} \in O(L)$. So $\sigma=\tau_{\sigma y-y}$ or $\tau_{\sigma y-y} \tau_{z}$.

When ord $\alpha>4$, put $\xi=1+\pi^{[(e-2) / 2]}$ and $u=\pi^{2} x-\pi^{2} y+\xi z$. Then

$$
Q(u)=\pi^{4}\left(1+\xi^{2}\right)+\pi^{2 e+3}-2 \pi^{4}=\pi^{4+2[(e-2) / 2]}+2 \pi^{4+[(e-2) / 2]}+\pi^{2 e+3} .
$$

So $\tau_{u} \in O(L)$ and $\tau_{u}(x)=x$. Write $\tau_{u} \sigma(y)=\alpha^{\prime} x+\beta^{\prime} y+\gamma^{\prime} z$. We can check ord $\alpha^{\prime} \leq 3$. Therefore we obtain the result as desired by the above arguments.

It is not difficult to check $Q(v) \in\left(1+p^{2}\right) \dot{F}^{2}$ for any maximal vector $v$ of $L$ which gives rise to a symmetry of $L$. So we obtain $\theta\left(O^{+}(L)\right) \subseteq\left(1+p^{2}\right) \dot{F}^{2}$.

Lemma 3. Suppose $s L \subseteq \vartheta$ and $\operatorname{rank} L \geq 4$ and $e \geq 3$. If $\operatorname{ord}(d L) \leq 7$ then $\theta\left(O^{+}(L)\right) \supseteq U \dot{F}^{2}$.

Proof. Using the above Lemma 2, considering components and dual lattices whenever necessary, there remain two cases to be treated.

(1) $L=L_{1} \perp L_{2}$ where $L_{1}$ is a binary unimodular lattice and $L_{2}$ is a binary $p^{2}$-modular lattice. Write $L_{1} \cong A\left(\varepsilon_{1} \pi^{r_{1}},-\varepsilon_{1}^{-1} \pi^{-r_{1}} \delta_{1}\right)$ with base $\left\{x_{1}, y_{1}\right\}$ and $0 \leq r_{1} \leq e$ and $D\left(1+\delta_{1}\right)=\delta_{1} \vartheta . L_{2} \cong \pi^{2} A\left(\varepsilon_{2} \pi^{r_{2}},-\varepsilon_{2}^{-1} \pi^{-r_{2}} \delta_{2}\right)$ with base $\left\{x_{2}, y_{2}\right\}$ and $0 \leq r_{2} \leq e$ and $D\left(1+\delta_{2}\right)=\delta_{2} \vartheta$.

By the same arguments as in Lemma 2, Case (4), and in [H, Lemma 1, Prop. C], and considering the dual lattice of $L$ if necessary, we only need to consider the case $0 \leq r_{1}=r_{2} \leq e-2$ and $\operatorname{ord}\left(-\varepsilon_{1}^{-1} \pi^{-r_{1}} \delta_{1}\right)>e$ and $\operatorname{ord}\left(-\varepsilon_{2}^{-1} \pi^{-r_{2}} \delta_{2}\right)>e$. Put $D\left(\varepsilon_{1} \varepsilon_{2}\right)=p^{t}$ with $1 \leq t \leq 2 e$ or $t=+\infty$.

If $t \leq e-r_{1}-1$, take $K=\vartheta x_{1} \perp \vartheta x_{2}$. Then $\theta\left(O^{+}(L)\right)=\dot{F}$ by the same arguments as in Lemma 2, Case(2).

If $t>e-r_{1}-1$, write $\varepsilon_{2}^{-1} \varepsilon_{1}=\xi^{2}+\sigma \pi^{t}$ where $\xi$ and $\sigma$ are in $U$.

When $e-r_{1}$ is odd, there exists $u$ in $\vartheta$ such that

$$
\begin{aligned}
\lambda+2 \pi^{r_{1}-e} \xi\left(\pi^{\left(e-r_{1}-1\right) / 2}-\xi\right) & \\
& -\sigma \pi^{t+r_{1}-e}-2 \varepsilon_{2}^{-1} \pi^{-e} u+\left(\varepsilon_{1} \varepsilon_{2}\right)^{-1} \pi^{-e-r_{1}} \delta_{1} u^{2}=0
\end{aligned}
$$

for any $\lambda \in \vartheta$ by Hensel's Lemma. Put $z=\pi x_{1}+\pi u y_{1}+\left(\pi^{\left(e-r_{1}-1\right) / 2}-\xi\right) x_{2}$ $\in L$. So $Q(z)=\varepsilon_{2} \pi^{e+1}(1+\lambda \pi)$ and $\tau_{z}$ is in $O(L)$. Therefore $\theta\left(O^{+}(L)\right) \supseteq$ $U \dot{F}^{2}$. 
When $e-r_{1}$ is even, there exists $u$ in $\vartheta$ such that

$$
\begin{aligned}
\lambda+2 \pi^{r_{1}+1-e} \xi\left(\pi^{\left(e-r_{1}-2\right) / 2}-\xi\right)-\sigma \pi^{t+r_{1}+1-e}-2 \varepsilon_{2}^{-1} \pi^{-e} u & \\
& +\left(\varepsilon_{1} \varepsilon_{2}\right)^{-1} \pi^{-r_{1}-e-1} \delta_{1} u^{2}=0
\end{aligned}
$$

for any $\lambda$ in $\vartheta$ by Hensel's Lemma provided ord $\left(-\varepsilon_{1}^{-1} \pi^{-r_{1}} \delta_{1}\right) \geq e+2$. Put $z=\pi x_{1}+u y_{1}+\left(\pi^{\left(e-r_{1}-2\right) / 2}-\xi\right) x_{2} \in L$. So $Q(z)=\varepsilon_{2} \pi^{e}(1+\lambda \pi)$ and $\tau_{z}$ is in $O(L)$. Therefore $\theta\left(O^{+}(L)\right) \supseteq U \dot{F}^{2}$.

Now we treat the case of $\operatorname{ord}\left(-\varepsilon_{1}^{-1} \pi^{-r_{1}} \delta_{1}\right)=e+1$. For any $\lambda$ in $U$, write

$$
\lambda\left(-\left(\varepsilon_{1} \varepsilon_{2}\right)^{-1} \pi^{-r_{1}-e-1} \delta_{1}\right)^{-1}=\alpha^{2}+\beta \pi^{d}
$$

with $\alpha, \beta \in U$ and $d \geq 1$. By Hensel's Lemma, there exists $u$ in $\vartheta$ such that

$$
\begin{aligned}
\left(\left(\varepsilon_{1} \varepsilon_{2}\right)^{-1} \pi^{-r_{1}-1-e} \delta_{1}\right)\left(\beta \pi^{d}\right)+ & \left(2 \varepsilon_{2}^{-1} \pi^{-e} \alpha-2 \pi^{\left(r_{1}-e\right) / 2} \xi\right) u \\
& +\left(\sigma \pi^{r_{1}+1+t-e}+2 \pi^{r_{1}+1-e} \xi^{2}\right) u^{2}=0 .
\end{aligned}
$$

Put $z=\pi u x_{1}+\alpha y_{1}+\left(\pi^{\left(e-r_{1}-2\right) / 2}-\xi u\right) x_{2} \in L$. So $Q(z)=\varepsilon_{2} \pi^{e}(1+\lambda \pi)$ and $\tau_{z}$ is in $O(L)$. Therefore $\theta\left(O^{+}(L)\right) \supseteq U \dot{F}^{2}$ by Lemma 1 .

(2) $L=L_{1} \perp L_{2}$ where $L_{1}$ is a binary unimodular lattice and $L_{2}$ is a binary $p^{3}$-modular lattice. Write $L_{1} \cong A\left(\varepsilon_{1} \pi^{r_{1}},-\varepsilon_{1}^{-1} \pi^{-r_{1}} \delta_{1}\right)$ with base $\left\{x_{1}, y_{1}\right\}$ and $0 \leq r_{1} \leq e$ and $D\left(1+\delta_{1}\right)=\delta_{1} \vartheta . L_{2} \cong \pi^{3} A\left(\varepsilon_{2} \pi^{r_{2}},-\varepsilon_{2}^{-1} \pi^{-r_{2}} \delta_{2}\right)$ with base $\left\{x_{2}, y_{2}\right\}$ and $0 \leq r_{2} \leq e$ and $D\left(1+\delta_{2}\right)=\delta_{2} \vartheta$. By the arguments similar to Lemma 2, Case (4), and [H, Lemma 1, Prop. C], and considering the dual lattice of $L$ if necessary, we only need to consider the case $0 \leq r_{1}$, $r_{2} \leq e-2 ; r_{1}=r_{2}+1$ or $r_{1}+1=r_{2}$; and $\operatorname{ord}\left(-\varepsilon_{1}^{-1} \pi^{-r_{1}} \delta_{1}\right)>e$ and $\operatorname{ord}\left(-\varepsilon_{2}^{-1} \pi^{-r_{2}} \delta_{2}\right)>e$.

When $r_{1}=r_{2}+1$, we can obtain the results as desired by the same arguments as in the above Case (1).

Now we treat the case $r_{2}=r_{1}+1$. Put $D\left(\varepsilon_{1} \varepsilon_{2}\right)=p^{t}$ with $1 \leq t \leq 2 e$ or $t=+\infty$.

If $t \leq e-r_{1}-2$, take $K=\vartheta x_{1} \perp \vartheta x_{2}$. Then $\theta\left(O^{+}(L)\right)=\dot{F}$ by the same arguments as in Lemma 2, Case (2).

If $t>e-r_{1}-2$, write $\varepsilon_{2}^{-1} \varepsilon_{1}=\xi^{2}+\sigma \pi^{t}$ where $\xi$ and $\sigma$ are in $U$.

When $e-r_{1}$ is even, there exists $u$ in $\vartheta$ such that

$$
\begin{aligned}
-\lambda-2 \pi^{r_{1}+1-e}\left(\pi^{\left(e-r_{1}-2\right) / 2}-1\right) & +\sigma \pi^{t-e+r_{1}+1} \xi^{-2}\left(\pi^{\left(e-r_{1}-2\right) / 2}-1\right)^{2} \\
& +2 \varepsilon_{2}^{-1} \pi^{-e} u-\varepsilon_{2}^{-2} \pi^{-\left(r_{1}+1\right)-e} \delta_{2} u^{2}=0
\end{aligned}
$$

for any $\lambda \in \vartheta$ by Hensel's Lemma. Put $z=\pi^{2} \xi^{-1}\left(\pi^{\left(e-r_{1}-2\right) / 2}-1\right) x_{1}+$ $x_{2}+u y_{2} \in L$. So $Q(z)=\varepsilon_{2} \pi^{e+2}(1+\lambda \pi)$ and $\tau_{z}$ is in $O(L)$. Therefore $\theta\left(O^{+}(L)\right) \supseteq U \dot{F}^{2}$.

When $e-r_{1}$ is odd, $r_{1}+1=r_{2} \leq e-2$ and $r_{1} \leq e-3$. Then there exists $u$ in $\vartheta$ such that 


$$
\begin{aligned}
\lambda+2 \pi^{-e+r_{1}+2}\left(\pi^{\left(e-r_{1}-3\right) / 2}-1\right)-\sigma \xi^{-2} \pi^{t-e+r_{1}+2}\left(\pi^{\left(e-r_{1}-3\right) / 2}-1\right)^{2} \\
-2 \pi^{-e} \varepsilon_{2}^{-1} u+\varepsilon_{2}^{-2} \pi^{-\left(r_{1}+1\right)} \delta_{2} \pi^{-e-1} u^{2}=0
\end{aligned}
$$

for any $\lambda \in \vartheta$ provided ord $\left(-\varepsilon_{2}^{-1} \pi^{-r_{2}} \delta_{2}\right) \geq e+2$. Put $z=\pi^{3}\left(\pi^{\left(e-r_{1}-3\right) / 2}\right.$ $-1) \xi^{-1} x_{1}+\pi x_{2}+u y_{2} \in L$. So $Q(z)=\varepsilon_{2} \pi^{e+3}(1+\lambda \pi)$ and $\tau_{z}$ is in $O(L)$. Therefore $\theta\left(O^{+}(L)\right) \supseteq U \dot{F}^{2}$.

Finally, we consider the case of $\operatorname{ord}\left(-\varepsilon_{2}^{-1} \pi^{-r_{2}} \delta_{2}\right)=e+1$. For any $\lambda \in U$, write $\lambda\left(-\varepsilon_{2}^{-2} \pi^{-\left(r_{1}+1\right)} \pi^{-e-1} \delta_{2}\right)^{-1}=\alpha^{2}+\beta \pi^{d}$ with $\alpha, \beta \in U$ and $d \geq 1$. By Hensel's Lemma, there exists $u$ in $\vartheta$ such that

$$
\begin{aligned}
\left(\varepsilon_{2}^{-2} \pi^{-\left(r_{1}+1\right)} \pi^{-e-1} \delta_{2}\right)\left(\beta \pi^{d}\right)+\sigma \xi^{-2} \pi^{t-1} & \\
& +\left(2 \varepsilon_{2}^{-1} \pi^{-e} \alpha-2 \pi^{\left(r_{1}+1-e\right) / 2}-2 \sigma \xi^{-2} \pi^{t+\left(r_{1}-e+1\right) / 2}\right) u \\
& +\left(2 \pi^{-e+r_{1}+2}+\sigma \xi^{-2} \pi^{t-e+r_{1}+2}\right) u^{2}=0 .
\end{aligned}
$$

Put $z=\pi^{3}\left(\pi^{\left(e-r_{1}-3\right) / 2}-u\right) \xi^{-1} x_{1}+\pi u x_{2}+\alpha y_{2} \in L$. So $Q(z)=$ $\varepsilon_{2} \pi^{e+3}(1+\lambda \pi)$ and $\tau_{z}$ is in $O(L)$. Therefore $\theta\left(O^{+}(L)\right) \supseteq U \dot{F}^{2}$ by Lemma 1 .

By the above lemmas and [X, Theorem 3.1] and the same arguments as in $[\mathrm{BD}]$ and by the results in $[\mathrm{X}]$ and $[\mathrm{EH}]$, we have If

TheOREm. Let $L$ be a regular $\vartheta$ lattice with $s L \subseteq \vartheta$ and $\operatorname{rank} L=n \geq 3$.

$$
\operatorname{ord}(d L)< \begin{cases}n(n-2) & \text { if } n \text { is even, } \\ (n-1)^{2} & \text { if } n \text { is odd }\end{cases}
$$

then $\theta\left(O^{+}(L)\right) \supseteq U \dot{F}^{2}$.

Remark. The bound on ord $(d L)$ in the above theorem is the best possible. For $e=1$ this easily follows from [EH, Theorem 3.14]. Consider the following example for $e \geq 2$ :

$$
L=\left\{\begin{array}{l}
A\left(1, \pi^{2 e-1}\right) \perp \pi^{4} A\left(1, \pi^{2 e-1}\right) \perp \ldots \perp \pi^{4(n / 2-1)} A\left(1, \pi^{2 e-1}\right) \\
\quad \text { with base }\left\{x_{1}, y_{1} ; x_{2}, y_{2} ; \ldots ; x_{n / 2}, y_{n / 2}\right\} \quad \text { if } n \text { is even, } \\
A\left(1, \pi^{2 e-1}\right) \perp \pi^{4} A\left(1, \pi^{2 e-1}\right) \perp \ldots \perp \pi^{4((n-1) / 2-1)} A\left(1, \pi^{2 e-1}\right) \\
\quad \perp\left\langle\pi^{2(n-1)}\right\rangle \\
\quad \text { with base }\left\{x_{1}, y_{1} ; x_{2}, y_{2} ; \ldots ; x_{(n-1) / 2}, y_{(n-1) / 2} ; z\right\} \quad \text { if } n \text { is odd. }
\end{array}\right.
$$

We will show that $\theta\left(O^{+}(L)\right) \subset U \dot{F}^{2}$.

First, by the same arguments as in the above Example when $e \geq 3$, and by the arguments as in [X, Example 4.3] when $e=2$, we can prove $O(L)$ is generated by the symmetries of $L$. Next we compute the spinor norms. For convenience, we only treat the case of even $n$. When $n$ is odd, the arguments are similar. 
When $e \geq 3$, we take any maximal vector $v$ of $L$ which gives rise to a symmetry of $L$. Write $v=\sum_{i=1}^{n / 2}\left(a_{i} x_{i}+b_{i} y_{i}\right)$. Then

$\begin{aligned}(*) \quad \operatorname{ord}(Q(v)) & =\operatorname{ord}\left(\sum_{i=1}^{n / 2} \pi^{4(i-1)}\left(a_{i}^{2}+2 a_{i} b_{i}+b_{i}^{2} \pi^{2 e-1}\right)\right) \\ & \leq e+\min _{1 \leq i \leq n / 2}\left\{4(i-1)+\operatorname{ord} a_{i}, 4(i-1)+\operatorname{ord} b_{i}\right\} .\end{aligned}$

We choose the largest $k$ such that

$$
\begin{aligned}
\min \left\{4(k-1)+\operatorname{ord} a_{k},\right. & \left.4(k-1)+\operatorname{ord} b_{k}\right\} \\
& =\min _{1 \leq i \leq n / 2}\left\{4(i-1)+\operatorname{ord} a_{i}, 4(i-1)+\operatorname{ord} b_{i}\right\} .
\end{aligned}
$$

If ord $a_{k} \leq 1$, then

$$
\begin{aligned}
\operatorname{ord}\left(\pi^{4(i-1)}\left(a_{i}^{2}+2 a_{i} b_{i}+b_{i}^{2} \pi^{2 e-1}\right)\right) & \\
& \quad \operatorname{ord}\left(\pi^{4(k-1)}\left(a_{k}^{2}+2 a_{k} b_{k}+b_{k}^{2} \pi^{2 e-1}\right)\right) \geq 2
\end{aligned}
$$

for all $i \neq k$ by $(*)$.

If ord $a_{k} \geq 2$, note that

$$
\begin{aligned}
Q(v)=\left(\sum_{i=1}^{n / 2} \pi^{2(i-1)} a_{i}\right)^{2}-2 & \sum_{\substack{1 \leq s<t \leq n / 2 \\
n / 2}} \pi^{2(s-1)+2(t-1)} a_{s} a_{t} \\
& \quad+\sum_{i=1}^{n / 2} b_{i}^{2} \pi^{4(i-1)+2 e-1}+2 \sum_{i=1}^{n / 2} a_{i} b_{i} \pi^{4(i-1)} .
\end{aligned}
$$

We have

$$
\begin{aligned}
& \operatorname{ord}(\left.-2 \pi^{2(s-1)+2(t-1)} a_{s} a_{t}\right)-\operatorname{ord} Q(v) \\
& \quad \geq e+2(s-1)+2(t-1)+\operatorname{ord} a_{s}+\operatorname{ord} a_{t}-\left(e+4(s-1)+\operatorname{ord} a_{s}\right) \\
& \quad=2(t-s)+\operatorname{ord} a_{t} \geq 2
\end{aligned}
$$

for any $1 \leq s<t \leq n / 2$ by $(*)$, and

$$
\begin{aligned}
& \operatorname{ord}\left(b_{i}^{2} \pi^{4(i-1)+2 e-1}\right)-\operatorname{ord} Q(v) \\
& \geq 2 \operatorname{ord} b_{i}+4(i-1)+(2 e-1)-\left(4(i-1)+\operatorname{ord} b_{i}+e\right) \\
& =\operatorname{ord} b_{i}+(e-1) \geq 2
\end{aligned}
$$

for any $1 \leq i \leq n / 2$ by $(*)$; also ord $a_{i} \geq 2$ for any $i \leq k$ by the choice of $k$.

So

$$
\begin{aligned}
& \operatorname{ord}\left(2 a_{i} b_{i} \pi^{4(i-1)}\right)-\operatorname{ord} Q(v) \\
& \quad \geq e+4(i-1)+\operatorname{ord} a_{i}+\operatorname{ord} b_{i}-\left(e+4(i-1)+\operatorname{ord} b_{i}\right)=\operatorname{ord} a_{i} \geq 2
\end{aligned}
$$

for any $i \leq k$ by $(*)$. 
Suppose there exists $j>k$ such that ord $a_{i}=\operatorname{ord} b_{i}=0$ and

$$
4(j-1)=\min \left\{4(k-1)+\operatorname{ord} a_{k}, 4(k-1)+\operatorname{ord} b_{k}\right\}+1 .
$$

Then

$$
\begin{aligned}
\operatorname{ord}\left(\pi^{4(i-1)}\left(a_{i}^{2}+2 a_{i} b_{i}+b_{i}^{2} \pi^{2 e-1}\right)\right) & \\
& \quad-\operatorname{ord}\left(\pi^{4(j-1)}\left(a_{j}^{2}+2 a_{j} b_{j}+b_{j}^{2} \pi^{2 e-1}\right)\right) \geq 2
\end{aligned}
$$

for any $i \neq j$ by $(*)$. Otherwise,

$$
\begin{aligned}
& \operatorname{ord}\left(2 a_{i} b_{i} \pi^{4(i-1)}\right)-\operatorname{ord} Q(v) \\
\geq & 4(i-1)+e+\operatorname{ord} a_{i}+\operatorname{ord} b_{i}-\left(e+\min \left\{4(k-1)+\operatorname{ord} a_{k}, 4(k-1)+\operatorname{ord} b_{k}\right\}\right) \geq 2
\end{aligned}
$$

for any $i>k$ by the choice of $k$.

Therefore we obtain $\theta\left(O^{+}(L)\right) \subseteq\left(1+p^{2}\right) \dot{F}^{2}$ by [H, Prop. D].

When $e=2$, the above arguments are still in force except

$$
\begin{aligned}
\operatorname{ord}\left(b_{i}^{2} \pi^{2 e-1} \pi^{4(i-1)}\right)-\operatorname{ord} Q(v) & \\
& \geq 2 \operatorname{ord} b_{i}+(2 e-1)+4(i-1)-\left(4(i-1)+\operatorname{ord} b_{i}+e\right) \\
& =e-1+\operatorname{ord} b_{i} \geq e-1=1 .
\end{aligned}
$$

Note that

$$
\begin{aligned}
Q(v)= & \left(\sum_{i=1}^{n / 2} \pi^{2(i-1)} a_{i}\right)^{2}+2\left(\sum_{i=1}^{n / 2} \pi^{2(i-1)} a_{i}\right)\left(\sum_{i=1}^{n / 2} \pi^{2(i-1)} b_{i}\right) \\
& +\left(\sum_{i=1}^{n / 2} \pi^{2(i-1)} b_{i}\right)^{2} \pi^{2 e-1}-2 \sum_{1 \leq s<t \leq n / 2} \pi^{2(s-1)+2(t-1)} a_{s} a_{t} \\
& -2 \sum_{1 \leq s<t \leq n / 2} \pi^{2(s-1)+2(t-1)} b_{s} b_{t} \pi^{2 e-1} \\
& -2 \sum_{1 \leq s \neq t \leq n / 2} \pi^{2(s-1)+2(t-1)} a_{s} b_{t} .
\end{aligned}
$$

We have

$$
\operatorname{ord}\left(2 \pi^{2(s-1)+2(t-1)} b_{s} b_{t} \pi^{2 e-1}\right)-\operatorname{ord} Q(v) \geq 2
$$

and

$$
\operatorname{ord}\left(2 \pi^{2(s-1)+2(t-1)} a_{s} b_{t}\right)-\operatorname{ord} Q(v) \geq 2
$$

for any $s \neq t$ by $(*)$. So we obtain $\theta\left(O^{+}(L)\right)=U \dot{F}^{2} \cap Q\left(\left[1, \dot{\pi}^{3}-1\right]\right)$ by $\left[\mathrm{X}_{0}\right]$ and [X, Remark 1].

By the above theorem, we can improve [BD, Prop. 4.1], in fact, we can modify $s_{p}(n)$ appearing there as follows:

$$
s_{p}(n)= \begin{cases}n(n-2) / 2 & \text { if } p \text { is nondyadic, } \\ s(n) & \text { if } p \text { is dyadic }\end{cases}
$$


where

$$
s(n)= \begin{cases}n(n-2) & \text { if } n \text { is even } \\ (n-1)^{2} & \text { if } n \text { is odd }\end{cases}
$$

\section{References}

[BD] W. R. Bon Durant, Spinor norms of rotations of local integral quadratic forms, J. Number Theory 33 (1989), 83-94.

[EH] A. G. Earnest and J. S. Hsia, Spinor norms of local integral rotations, II, Pacific J. Math. 61 (1975), 71-86; errata 115 (1984), 493-494.

[H] J. S. Hsia, Spinor norms of local integral rotations, I, ibid. 57 (1975), 199-206.

[K] M. Kneser, Klassenzahlen indefiniter quadratischer Formen in drei oder mehr Veränderlichen, Arch. Math. (Basel) 7 (1956), 323-332.

[O] O. T. O'Meara, Introduction to Quadratic Forms, Springer, New York 1963.

$\left[\mathrm{X}_{0}\right] \mathrm{F} . \mathrm{Xu}$, A remark on spinor norms of local integral rotations, I, Pacific J. Math. 136 (1989), 81-84.

[X] - Integral spinor norms in dyadic local fields, I, ibid., to appear.

DEPARTMENT OF MATHEMATICS

UNIVERSITY OF SCIENCE AND TECHNOLOGY OF CHINA

HEFEI, ANHUI 230026

PEOPLE'S REPUBLIC OF CHINA

Received on 26.3.1991

and in revised form on 9.8.1991 and 3.8.1992 